\title{
The role of intravascular ultrasound in the treatment of chronic total occlusion with percutaneous coronary intervention
}

\author{
Kambis Mashayekhi ${ }^{1}$, Michael Behnes ${ }^{2}$ \\ ${ }^{1}$ Division of Cardiology and Angiology II, University Heart Center Freiburg, Bad Krozingen, Germany \\ ${ }^{2}$ First Department of Medicine, University Medical Centre Mannheim (UMM), Faculty of Medicine \\ Mannheim, University of Heidelberg, European Center for AngioScience (ECAS), and DZHK \\ (German Center for Cardiovascular Research) partner site Heidelberg/Mannheim, Mannheim, Germany
}

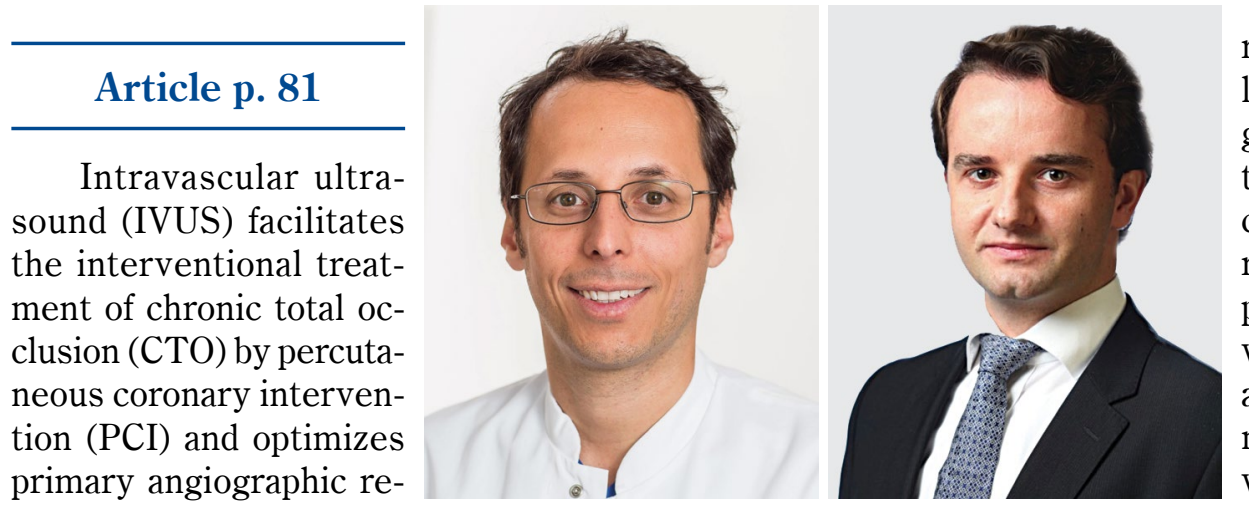
sults with relevant clinical impact $[1,2]$. There are several indications, where IVUS may be useful during CTO-PCI: 1) to define the entry point of an ambiguous proximal cap; 2) to simplify the reverse controlled antegrade-retrograde tracking (rCART) maneuver; 3 ) for IVUS controlled antegrade reentry techniques; and 4) finally for optimizing the primary result after stent implantation. Focusing on the rCART maneuver, there are four potential scenarios of antegrade and retrograde wire position: 1) antegrade and retrograde wires are in the intraplaque position; or 2) in the subintimal position. In both situations the connection of both wires can mostly be performed with a polymer-jacket lower gram tip-loaded wire after antegrade balloon inflation and facilitated with a mother-in-child catheter. Further possible scenarios are; 3 ) when the antegrade wire is intraplaque and the retrograde wire is subintimal; or 4) vice versa. Once the antegrade wire has entered the intraplaque position, antegrade balloon angioplasty may be very helpful for reconnection, nevertheless a penetrative higher gram tip-loaded wire is often mandatory. The most complex rCART scenario represents the subintimal position of the antegrade wire, especially after creating antegrade hematoma, and the retrograde wire is intraplaque. In this setting the antegrade dilatation of the subintimal space is often useless, since the external elastic lamina is compressed from the subintimal balloon inflation, followed by an immediate collapse of the subintimal space. This may even cause further enlargement of antegrade hematoma after multiple balloon dilatations, reducing the chance for reconnecting both wires prior to externalization. Therefore, IVUS guidance is specifically recommended after rCART failure to define another level of reconnection for antegrade and retrograde wires during retrograde CTO-PCI.

In this issue of 'Cardiology Journal', Chu et al. [3] evaluated the usage of high definition intravascular ultrasound (HD-IVUS) with a $60 \mathrm{MHz}$ catheter to understand the position of the antegrade and retrograde wire during rCART maneuver. In their particular case the anatomy of the vessel during rCART appeared divided in two halfs reflecting the characteristic 'yin-yang' sign: One half dark due to intraplaque wire position, and the other whitish due to subintimal hematoma. This distinctive IVUS

Address for correspondence: Dr. Kambis Mashayekhi, Division of Cardiology and Angiology II, University Heart Center Freiburg, Bad Krozingen, Germany, e-mail: Kambis.Mashayekhi@universitaets-herzzentrum.de 
sign is easy to remember. It is important to understand that the 'ying-yang' sign may affect rCART failure. Once the antegrade wire (IVUS probe) is in the subintimal (whitish half) and the retrograde wire is in the intraplaque space (dark half), using HD-IVUS revealed another reconnection scenario more proximally to the 'ying-yang' sign.

The clear understanding of IVUS imaging during CTO-PCI is an essential diagnostic tool to decrease complications, while improving both success rates and both short- and long-term PCI results. Implementation of IVUS use should be recommended to all CTO-PCI operators.

Conflict of interest: None declared

\section{References}

1. Kim BK, Shin DH, Hong MK, et al. Clinical impact of intravascular ultrasound-guided chronic total occlusion intervention with zotarolimus-eluting versus biolimus-eluting stent implantation: randomized study. Circ Cardiovasc Interv. 2015; 8(7): e002592, doi: $10.1161 /$ CIRCINTERVENTIONS.115.002592, indexed in Pubmed: 26156151.

2. Kim D, Hong SJ, Kim BK, et al. Outcomes of stent optimisation in intravascular ultrasound-guided intervention for long or chronic totally occluded coronary lesions. EuroIntervention. 2019 [Epub ahead of print], doi: 10.4244/EIJ-D-19-00762, indexed in Pubmed: 31829943.

3. Chu M, Martínez-Hervás-Alonso MA, Reisbeck B, et al. The yin-yang sign in the detection of subintimal hematoma with high-definition intravascular ultrasound. Cardiol J. 2020; 27(1): 81-82, doi: 10.5603/CJ.2020.0115. 